\title{
Inorganic Nanoribbons with Unpassivated Zigzag Edges: Half Metallicity and Edge Reconstruction
}

\author{
Menghao $\mathrm{Wu}^{1}$, Xiaojun $\mathrm{Wu}^{2}$, Yong $\mathrm{Pei}^{3}$, and Xiao Cheng Zeng ${ }^{1}(\varangle)$ \\ ${ }^{1}$ Department of Chemistry and Department of Physics and Astronomy, University of Nebraska-Lincoln, Lincoln, Nebraska 68588, USA \\ ${ }^{2}$ Department of Materials of Science and Engineering, Hefei National Laboratory for Physical Materials at Microscale, University of Science \\ and Technology of China, Hefei, Anhui 230026, China \\ ${ }^{3}$ Key Laboratory of Environmentally Friendly Chemistry and Application of Ministry of Education, Xiangtan University, Xiangtan 411105, China \\ Received: 21 September 2010 / Revised: 2 November 2010 / Accepted: 5 November 2010 \\ (C) The Author(s) 2010. This article is published with open access at Springerlink.com
}

\begin{abstract}
We have investigated the electronic and structural properties of inorganic nanoribbons (BN, AlN, GaN, SiC, and $\mathrm{ZnO}$ ) with unpassivated zigzag edges using density functional theory calculations. We find that, in general, the unpassivated zigzag edges can lead to spin-splitting of energy bands. More interestingly, the inorganic nanoribbons AlN and $\mathrm{SiC}$ with either one or two edges unpassivated are predicted to be half metallic. Possible structural reconstruction at the unpassivated edges and its effect on the electronic properties are investigated. The unpassivated $\mathrm{N}$ edge in the BN nanoribbon and $\mathrm{P}$ edge in the AlP nanoribbon are energetically less stable than the corresponding reconstructed edge. Hence, edge reconstruction at the two edges may occur at high temperatures. Other unpassivated edges of the inorganic nanoribbons considered in this study are all robust against edge reconstruction.
\end{abstract}

\section{KEYWORDS}

AlN and SiC nanoribbons, half metallicity, unpassivated zigzag edge, edge reconstruction, density functional theory

\section{Introduction}

Graphene, a two-dimensional (2D) material, has attracted considerable research attention due to its unique physical properties such as massless Dirac fermion behavior [1-3], room-temperature quantum Hall effect, $[4,5]$ high carrier mobility and thermal conductivity, and high electronic coherence [6]. Moreover, when a quasi-one-dimensional (Q1D) nanoribbon is cut out from the $2 \mathrm{D}$ graphene sheet, the energy band gap of the graphene nanoribbon (GNR) is no longer zero but dependent on the width and crystallographic orientation of the GNR [7-12].
In particular, zigzag-edged GNRs (zGNRs) show distinct electronic properties due to their special localized edge states which are antiferromagnetically coupled [11]. Recent theoretical studies suggest that half metallicity can be also achieved in zGNRs [13-17]. If confirmed, the half metallicity could be exploited for future spintronic applications because the electric current can be fully spin polarized when going through a half metallic system [18-20].

In addition to GNRs, some other Q1D inorganic structures such as $\mathrm{BN}, \mathrm{AlN}, \mathrm{GaN}, \mathrm{SiC}$, and $\mathrm{ZnO}$ nanotubes and nanoribbons have been investigated by theoretical means [21-26]. In Ref. [21], Zheng et al.

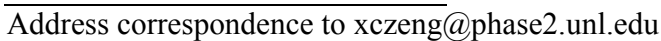


showed from first-principles calculations that the zigzag BN nanoribbon (zBNNR) can be half metallic if its $\mathrm{N}$ edge is unpassivated. It would be interesting to know whether the same electronic behavior can be seen in other inorganic nanoribbons with unpassivated edges. Nevertheless, unpassivated edges may be very unstable and, if so, edge reconstruction may take place, akin to structural reconstruction of the unpassivated edges of GNRs [27]. In this article, we present calculated electronic structures of a number of inorganic zigzag nanoribbons, including the main group III nitrides, the group III phosphides, $\mathrm{SiC}$, and $\mathrm{ZnO}$, based on density functional theory (DFT) calculations. We find that some of these nanoribbons with either one or two unpassivated edges can be half metallic. Possible edge reconstruction on all unpassivated edges is also investigated in detail. Some of the unpassivated edges are quite unstable, and edge reconstructions can take place above room temperature.

\section{Computational methods}

The DFT calculations were carried out using the $\mathrm{DMol}^{3}$ package [28-30]. The generalized gradient approximation (GGA) in the Perdew-Burke-Ernzerhof (PBE) form, together with the all-electron double numerical (DNP) basis set with polarized functions were chosen for the spin-unrestricted DFT computations [31]. The real-space global cutoff radius was set to be $3.7 \AA$. To simulate the inorganic nanoribbons, a rectangular supercell with dimensions $36 \times L \times 16 \AA^{3}$ was built, where $L$ was chosen to be twice the width of the hexagonal ring and hence depended on the nanoribbon concerned. The nearest distance between the nanoribbon and its nearest image (under periodic boundary conditions) was greater than $16 \AA$. For geometric optimization, the Brillouin zone was sampled with $1 \times 20 \times 1 k$ points using the Monkhorst-Pack scheme [32]. After geometric optimization, the forces on all atoms were less than $0.0002 \mathrm{Ha} / \AA$. To further confirm the predicted half metallicity for certain inorganic nanotubes, we re-examined their band structures using the screened exchange hybrid Heyd-ScuseriaErnzerhof (HSE) [33, 34] functional and the 6-31G basis set (implemented in the Gaussian 09 package [35]).

\section{Results and discussion}

\subsection{Half metallicity}

We use the number of parallel zigzag chains $n$ in a zigzag-edged nanoribbon to describe the width of the nanoribbon. Here, $n=8$ was chosen for all the nanoribbons considered. First, we focus on two group III nitrides, AlN and GaN. In the zigzag-edged AlN nanoribbon (zAlNNR), all the outermost atoms at the $\mathrm{Al}$ edge are $\mathrm{Al}$ atoms while all the outermost atoms at the opposing edge (i.e., the $\mathrm{N}$ edge) are $\mathrm{N}$ atoms. As shown in Fig. 1(a), when the Al edge of a zAlNNR is passivated by hydrogen atoms (designated as zAlNNR-HAl), the calculated band structure suggests that the zAlNNR-HAl is a spin-polarized semiconductor with a band gap of $\sim 0.2 \mathrm{eV}$. When the $\mathrm{H}$ atoms on the $\mathrm{Al}$ edge are removed, however, the calculated band structure suggests that the pristine zAlNNR is a half metal, which is also confirmed by DFT calculations using the HSE functional. As can be seen in Fig. 1(b), the $\mathrm{N}$ edge is ferromagnetic while the $\mathrm{Al}$ edge is antiferromagnetic, similar to the pristine zBNNR [19]. For zGaNNR-HGa, the $\mathrm{N}$ atoms at the $\mathrm{N}$-edge no longer lie on a single straight line along the ribbon direction, but become slightly modulated in a periodic fashion as shown in Fig. 1(c). The calculated band structure suggests that it is a spin-polarized semiconductor. When the $\mathrm{H}$ atoms on the $\mathrm{Ga}$ edge are removed, it is still a semiconductor, although the band gap is reduced to $0.05 \mathrm{eV}$ as shown in Fig. 1(d).

For group III phosphides, we consider BP and AlP nanoribbons. As shown in Fig. 2(a), the band structure calculations suggest zBPNR-HB is metallic if the $\mathrm{P}$ edge is unpassivated. For zAlPNR-HAl, the P edge is antiferromagnetic and the system is a spin-unpolarized semiconductor as shown in Fig. 2(b). Besides the group III/ $\mathrm{V}$ compounds, we also consider $\mathrm{SiC}$ and $\mathrm{ZnO}$ nanoribbons. As shown in Fig. 2(c), when the Si edge of $\mathrm{zSiCNR}-\mathrm{HC}$ is unpassivated, the spin distribution at the two edges in the ground state is ferromagnetically coupled. The nanoribbon is predicted to be a half metal based on the calculated band structure using both the PBE and HSE functionals. For zZnONR-HZn, shown in Fig. 2(d), the spin distribution at the two edges is antiferromagnetically coupled, and the 

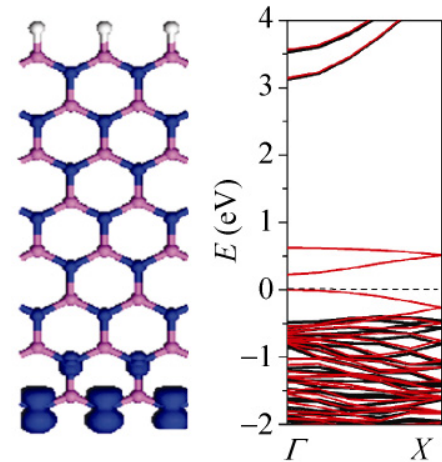

(a)

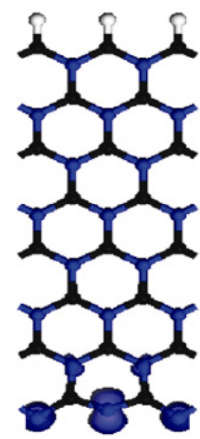

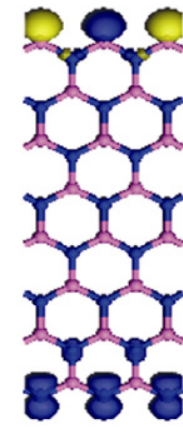

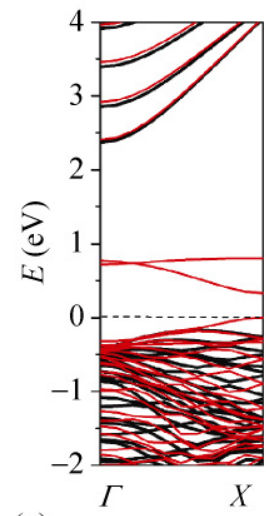

(c)
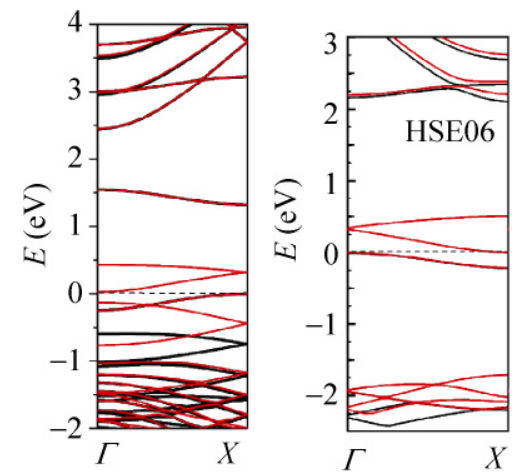

(b)

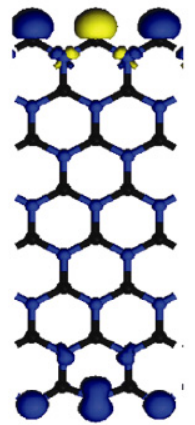

(d)

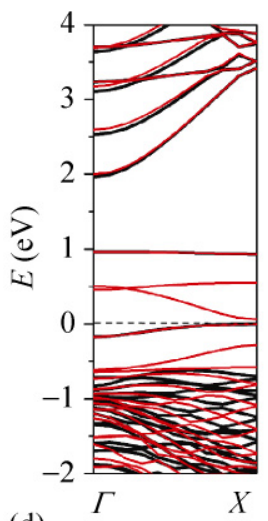

Figure 1 The spin density (left panel) and spin-polarized band structures (right panel) of (a) zAlNNR-HAl, (b) pristine zAlNNR, (c) zGaNNR-HGa, and (d) pristine zGaNNR. Small balls in white, pink, blue, and black denote hydrogen, aluminum, nitrogen, and gallium atoms, respectively. For spin density, blue and yellow lobes represent spin-up and spin-down regions, respectively; and the range of the iso-values of spin density is $[-0.03,0.03] \mathrm{e} / \AA^{3}$. In the band structure graphs, black represents the spin-up channel and red represents the spin-down channel. The Fermi level is denoted by the horizontal dashed line

nanoribbon is metallic. If all the $\mathrm{H}$ atoms at the $\mathrm{Zn}$ edge are removed, the nanoribbon is still metallic, but also becomes ferromagnetic as shown in Fig. 2(e). Such ferromagnetism has also been recently predicted to appear in $\mathrm{ZnO}$ nanowires [36].

\subsection{Edge reconstruction}

According to Ref. [27], in zGNRs, the reconstruction of an unpassivated zigzag edge may spontaneously take place at room temperature, where two neighboring hexagons can transform into a pentagon and heptagon. Here we investigate a similar edge reconstruction that may take place at the unpassivated edges of any of the inorganic nanoribbons discussed above. First, we consider the half metallic zBNNR-HB reported in Ref. [21]. As shown in Figs. 3(a)-3(c), the $N$ edge has two ways of reconstruction: (1) it can reconstruct into a symmetric structure as shown in Fig. 3(b), where the pentagon is formed by two $\mathrm{N}$ atoms and three $\mathrm{B}$ atoms. This way of edge reconstruction is denoted as the A style; (2) The $\mathrm{N}$ edge may also reconstruct into an unsymmetrical structure, as shown in Fig. 3(c), where each pentagon is formed by three $\mathrm{N}$ atoms and two $\mathrm{B}$ atoms. This way of edge reconstruction is denoted as the B style. After the edge reconstruction in the A style, the system is $1.06 \mathrm{eV} /$ supercell higher in energy, while in the B style, the energy is lowered by $0.22 \mathrm{eV} /$ supercell. Hence, the B style is energetically more favorable. Our calculations, using the nudged elastic band method $[37,38]$ implemented in $\mathrm{DMol}^{3}$, also showed that the activation barrier for the edge reconstruction in the B style is about $1.0 \mathrm{eV}$. Quantum molecular dynamics simulations (for $5 \mathrm{ps}$ ) of this system at $800 \mathrm{~K}$ were performed and no sign of spontaneous reconstruction of the $\mathrm{N}$ edge was observed, suggesting the edge reconstruction would occur at a very high temperature.

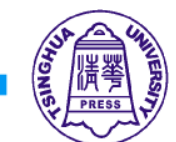



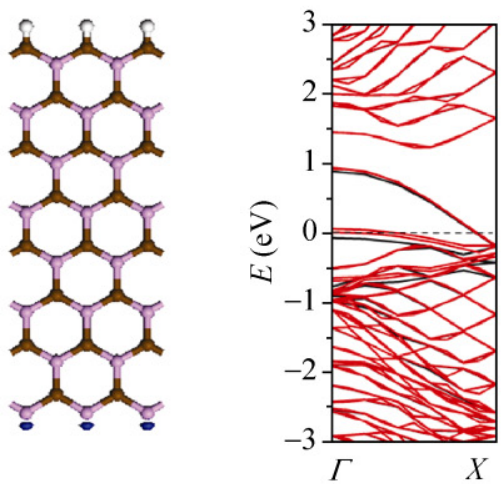

(a)
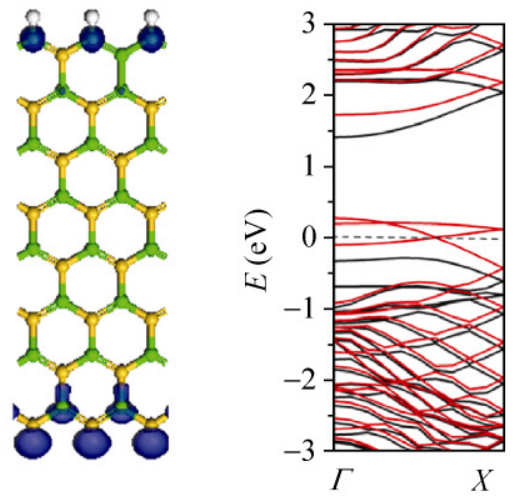

(c)

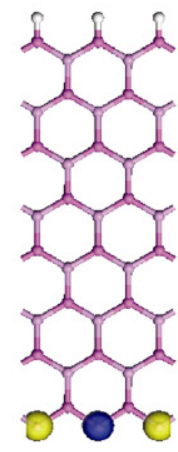

(b)
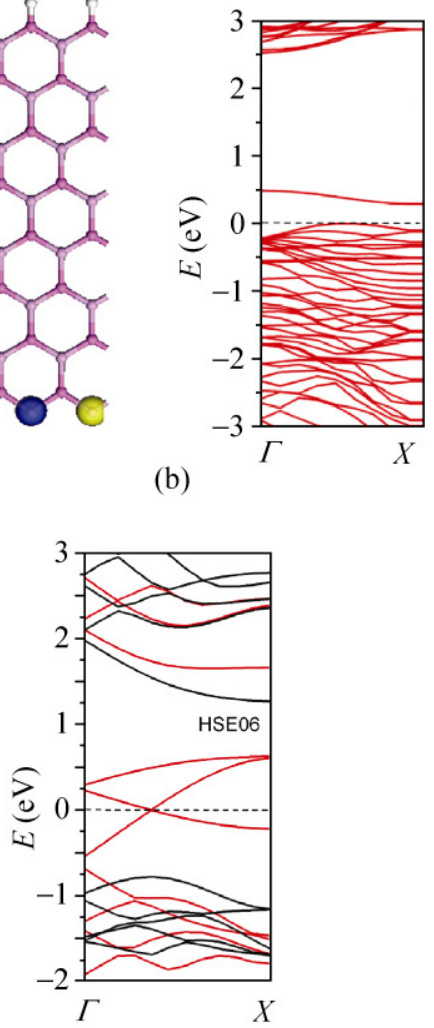

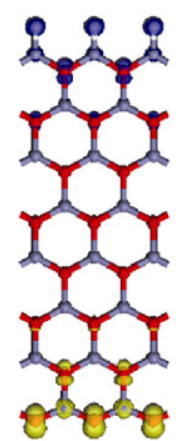

(d)

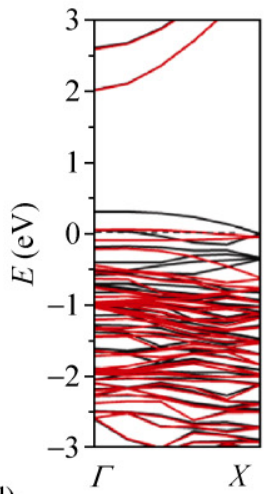

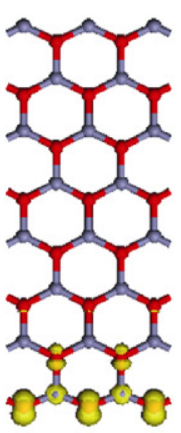

(e)

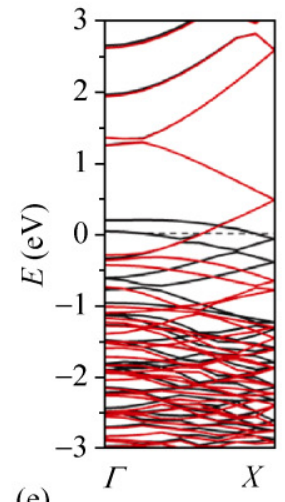

Figure 2 The spin density and spin-polarized band structures of (a) zBPNR-HB, (b) zAlPNR-HAl, (c) zSiCNR-HC, (d) zZnONR-HZn, and (e) pristine zZnONR. Small balls in green, white, pink, blue, black, brown, purple, yellow, grey, and red denote carbon, hydrogen, aluminum, nitrogen, gallium, boron, phosphorus, silicon, zinc, and oxygen atoms, respectively. For spin density, blue, and yellow lobes represent spin-up and spin-down regions, respectively; the range of the iso-values of spin density is $[-0.03,0.03] \mathrm{e} / \AA^{3}$. In the band structure graphs, black represents the spin-up channel and red represents the spin-down channel. The Fermi level is denoted by the horizontal dashed line

The calculated band structure for the reconstructed nanoribbon (in the B style) is shown in Fig. 3(d). Interestingly, after edge reconstruction, the nanoribbon is no longer a half metal but becomes a wide-gap semiconductor.

For the pristine zAlNNR, we first consider reconstruction of the $\mathrm{Al}$ edge. The edge reconstructions in the A and B styles are shown in Figs. 4(a) and 4(b), respectively. The calculations show that after edge reconstruction in the A or B style the energy will be raised by $3.4 \mathrm{eV}$ or $1.4 \mathrm{eV}$, respectively. Hence, the unpassivated $\mathrm{Al}$ edge appears to be robust against edge reconstruction. Figures 4(c) and 4(d) display the edge reconstruction at the $\mathrm{N}$ edge in the $\mathrm{A}$ and $\mathrm{B}$ style, 


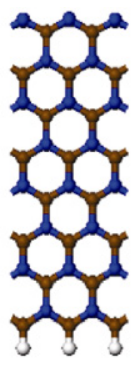

(a)

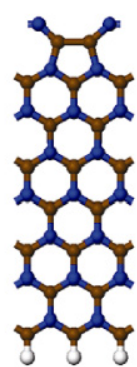

(b)

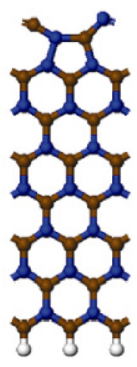

(c)

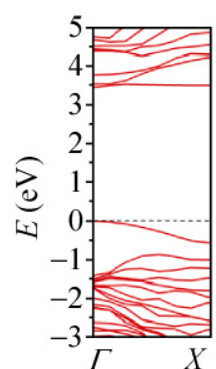

(d)
Figure 3 The optimized structures of zBNNR-HB (a) before, and after the edge reconstruction in (b) the A style and (c) B style at the $\mathrm{N}$ edge. White, blue, and brown balls denote hydrogen, nitrogen, and boron atoms, respectively. The band structure of nanoribbon (c) is shown in $(\mathrm{d})$

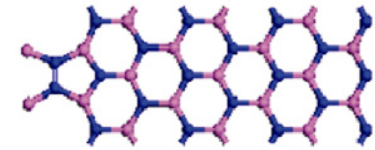

(a)

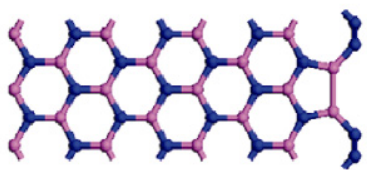

(c)

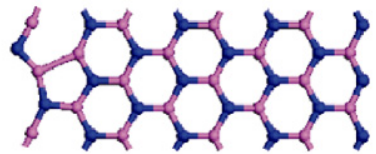

(b)

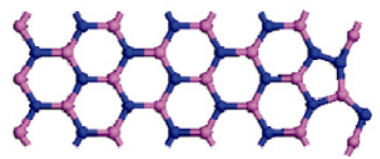

(d)
Figure 4 The optimized structures of zAlNNR after edge reconstruction in (a) the A style and (b) the B style at the Al edge, and in (c) the A style and (d) the B style after reconstruction at the $\mathrm{N}$ edge. Blue and pink balls denote nitrogen and aluminum atoms, respectively

respectively. Our calculations show that upon edge reconstruction in the A style, the energy will be lowered by $0.26 \mathrm{eV}$ while in the $\mathrm{B}$ style it will be increased by $0.64 \mathrm{eV}$. However, even though the edge reconstruction in the A style is energetically favorable, the activation barrier for the edge reconstruction is typically very high $(\sim 3.5 \mathrm{eV})$. Hence, it is unlikely for an $\mathrm{N}$ atom at the edge to swap position with its nearby $\mathrm{Al}$ atom. As a consequence, the unpassivated $\mathrm{N}$ edge can still be considered as being very stable against edge reconstruction.
In Table 1, we list the energy changes for edge reconstruction in the $\mathrm{A}$ and $\mathrm{B}$ styles at the unpassivated edge in zGaNNR-HGa, zBPNR-HB, zAlPNR-HAl, zSiCNR-HC, and zZnONR-ZnH. For zGaNNR-HGa, the pentagon and heptagon are not stable after edge reconstruction in the style $\mathrm{A}$ at the $\mathrm{N}$ edge because the $\mathrm{N}$ atoms will be converted into free $\mathrm{N}_{2}$ molecules, as shown in Fig. 5. The energy change for the edge reconstruction of $\mathrm{zZnONR}-\mathrm{HZn}$ in the $\mathrm{B}$ style is not given in Table 1 , since the unpassivated $O$ edge recovers its original zigzag structure after the geometric optimization, when the edge reconstruction is initially imposed on it.

As shown in Table 1, only the energy of zAlPNR$\mathrm{HAl}$ is lowered on edge reconstruction in the B style. Moreover, our calculations show that the activation barrier for edge reconstruction in this case is about $0.65 \mathrm{eV}$, implying that spontaneous edge reconstruction is feasible at room temperature. The normal bond lengths of $\mathrm{Al}-\mathrm{Al}, \mathrm{Al}-\mathrm{P}$, and $\mathrm{P}-\mathrm{P}$ are $2.22 \AA, 2.21 \AA$, and $2.20 \AA$, respectively, which are nearly the same. This observation is similar to the case of zBNNR-HB where the normal bond lengths of $\mathrm{B}-\mathrm{B}, \mathrm{B}-\mathrm{N}$, and $\mathrm{N}-\mathrm{N}$ are nearly the same $(1.42 \AA, 1.45 \AA$, and $1.48 \AA$, respectively). Considering the edge reconstruction of GNR reported in Ref. [27], it seems that edge reconstruction in the $\mathrm{B}$ style tends to occur at the unpassivated edge with two types of constituent atoms giving rise to nearly the same bond lengths between them.

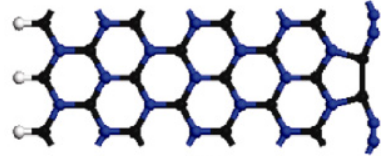

(a)

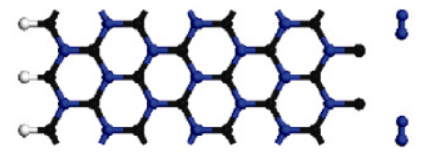

(b)
Figure 5 For the edge reconstruction of (a) zGaNNR-HGa in the A style, (b) the edge $\mathrm{N}$ atoms form free $\mathrm{N}_{2}$ molecules after geometric optimization. White, blue, and black balls denote the hydrogen, nitrogen, and gallium atoms, respectively

Table 1 Energy changes associated with edge reconstruction in the A and B styles at the unpassivated edge in zGaNNR-HGa zBPNR-HB, zAlPNR-HAl, zSiCNR-HC, and zZnONR-HZn

\begin{tabular}{cccccc}
\hline Reconstruction & zGaNNR-HGa & zBPNR-HB & zAlPNR-HAl & zSiCNR-HC & zZnONR-HZn \\
\hline A style $(\mathrm{eV})$ & -2.92 & 1.03 & 0.18 & 1.19 & 2.13 \\
B style $(\mathrm{eV})$ & 0.11 & 1.00 & -0.015 & 1.35 & N/A \\
\hline
\end{tabular}




\section{Conclusions}

We have shown that the unpassivated zigzag edges of the inorganic nanoribbons $(\mathrm{BN}, \mathrm{AlN}, \mathrm{GaN}, \mathrm{SiC}$, and $\mathrm{ZnO}$ ) can lead to spin-splitting energy bands in general. More interestingly, the inorganic nanoribbons $\mathrm{AlN}$ and $\mathrm{SiC}$ with either one or two edges unpassivated are predicted to be half metals. Possible structural reconstruction at the unpassivated edges and its effect on the electronic properties have been investigated. The unpassivated $\mathrm{N}$ edge in the $\mathrm{BN}$ nanoribbon and the $\mathrm{P}$ edge in the AlP nanoribbon are energetically less stable compared to the reconstructed edge. Hence, edge reconstruction is possible at high temperatures. Other unpassivated edges in the inorganic nanoribbons considered here are robust against edge reconstruction.

\section{Acknowledgements}

This work was supported by grants from the National Science Foundation (NSF) (No. DMR-0820521), NSF (No. CMMI-0709333), and the Nebraska Research Initiative, and by the University of Nebraska's Holland Computing Center.

Open Access: This article is distributed under the terms of the Creative Commons Attribution Noncommercial License which permits any noncommercial use, distribution, and reproduction in any medium, provided the original author(s) and source are credited.

\section{References}

[1] Novoselov, K. S.; Geim, A. K.; Morozov, S. V.; Jiang, D.; Katsnelson, M. I.; Grigorieva, I. V.; Dubonos, S. V.; Firsov, A. A. Two-dimensional gas of massless Dirac fermions in graphene. Nature 2005, 438, 197-200.

[2] Katsnelson, M. I.; Novoselov, K. S.; Geim, A. K. Chiral tunnelling and the Klein paradox in graphene. Nat. Phys. 2006, 2, 620-625.

[3] Zhou, S. Y.; Gwenon, G. H.; Graf, J.; Ferdorov, A. V.; Spataru, C. D.; Diehl, R. D.; Kopelevich, Y.; Lee, D. H.; Louie, S. G.; Lanzara, A. First direct observation of Dirac fermions in graphite. Nat. Phys. 2006, 2, 595-599.

[4] Novoselov, K. S.; Jiang, Z.; Zhang, Y.; Morozov, S. V.; Stormer, H. L.; Zeitler, U.; Maan, J. C.; Boebinger, G. S.;
Kim, P.; Geim, A. K. Room-temperature quantum Hall effect in graphene. Science 2007, 315, 1379.

[5] Zheng, Y.; Ando, T. Hall conductivity of a two-dimensional graphite system. Phys. Rev. B 2002, 65, 245420.

[6] Novoselov, K. S.; Geim, A. K.; Morozov, S. V.; Jiang, D.; Zhang, Y.; Dubonos, S. V.; Grigonrieva, I. V.; Firsov, A. A. Electric field effect in atomically thin carbon films. Science 2004, 306, 666-669.

[7] Fujita, M.; Wakabayasi, K.; Nakada, K.; Kusakabe, K. Peculiar localized state at zigzag graphite edge. J. Phys. Soc. Jpn. 1996, 65, 1920-1923.

[8] Nakada, K.; Fujita, M.; Dresselhaus, G.; Dresselhaus. M. S. Edge state in graphene ribbons: Nanometer size effect and edge shape dependence. Phys. Rev. B 1996, 54, 17954 -17961.

[9] Miyamoto, Y.; Nakada, K.; Fujita, M. First-principles study of edge states of H-terminated graphitic ribbons. Phys. Rev. B 1999, 60, 16211-16211.

[10] Kusakabe, K.; Maruyama, M. Magnetic nanographite. Phys. Rev. B 2003, 67, 092406.

[11] Pisani, L.; Chan, J. A.; Montanari, B.; Harrison, N. M. Electronic structure and magnetic properties of graphitic ribbons. Phys. Rev. B 2007, 75, 064418.

[12] Son, Y. W.; Cohen, M. L.; Louie, S. G. Energy gaps in graphene nanoribbons. Phys. Rev. Lett. 2006, 97, 216803.

[13] Son, Y. W.; Cohen, M. L.; Louie, S. G. Half-metallic graphene nanoribbons. Nature 2006, 444, 347-349.

[14] Kan, E. J.; Li, Z.; Yang, J. L.; Hou, J. G. Will zigzag graphene nanoribbon turn to half metal under electric field? Appl. Phys. Lett. 2007, 91, 243116.

[15] Kan, E. J.; Li, Z.; Yang, J. L.; Hou, J. G. Half-metallicity in edge-modified zigzag graphene nanoribbons. J. Am. Chem. Soc. 2008, 130, 4224-4225.

[16] Wu, M. H.; Wu, X.; Zeng, X. C. Exploration of half metallicity in edge-modified graphene nanoribbons. J. Phys. Chem C. 2010, 114, 3937-3944.

[17] Wu, M.; Wu, X.; Gao, Y.; Zeng, X. C. Materials design of half metallic graphene and graphene nanoribbons. Appl. Phys. Lett. 2009, 94, 223111.

[18] de Groot, R. A.; Mueller, F. M.; van Engen, P. G.; Buschow, K. H. J. New class of materials: Half-metallic ferromagnets. Phys. Rev. Lett. 1983, 50, 2024-2027.

[19] Prinz, G. A. Magnetoelectronics. Science 1998, 282, 16601663.

[20] Ziese, M. Extrinsic magnetotransport phenomena in ferromagnetic oxides. Rep. Prog. Phys. 2002, 65, 143-249.

[21] Zheng, F.; Zhou, G.; Liu, Z.; Wu, J.; Duan, W.; Gu, B. L.; Zhang, S. B. Half metallicity along the edge of zigzag boron nitride nanoribbons. Phys. Rev. B 2008, 78, 205415.

[22] Botello-Mendez, A. R.; Lopez-Urias, F.; Terrones, M.; 
Terrones, H. Magnetic behavior in zinc oxide zigzag nanoribbons. Nano. Lett. 2008, 8, 1562-1565.

[23] Lee, S. M.; Lee, Y. H.; Hwang, Y. G.; Elsner, J.; Porezag, D.; Frauenheim, T. Stability and electronic structure of GaN nanotubes from density-functional calculations. Phys. Rev. B 1999, 60, 7788-7791.

[24] Du, A. J.; Zhu, Z. H.; Chen, Y.; Lu, G. Q.; Smith, S. C. First principle studies of zigzag AlN nanoribbon. Chem. Phys. Lett. 2009, 469, 183-185.

[25] Sun, L.; Li, Y.; Li, Z.; Li, Q.; Zhou, Z.; Chen, Z.; Yang, J. L.; Hou, J. G. Electronic structures of SiC nanoribbons. $J$. Chem. Phys. 2008, 129, 174114.

[26] Pan, H.; Feng, Y. F. Semiconductor nanowires and nanotubes: Effects of size and surface-to-volume ratio. ACS Nano 2008, 2, 2410-2414.

[27] Koskinen, P.; Malola, S.; Hakkinen, H. Self-passivating edge reconstructions of graphene. Phys. Rev. Lett. 2008, 101, 115502 .

[28] Delley, B. An all-electron numerical method for solving the local density functional for polyatomic molecules. J. Chem. Phys. 1990, 92, 508-517.

[29] Delley, B. From molecules to solids with the DMol3 approach. J. Chem. Phys. 2000, 113, 7756-7764.

[30] $\mathrm{Dmol}^{3} 4.4$ is a density functional theory quantum mechanical package available from Accelrys Software Inc.

[31] Perdew, J. P.; Burke, K.; Ernzerhof, M. Generalized gradient approximation made simple. Phys. Rev. Lett. 1996, 77,
3865-3868.

[32] Monkhorst, H. J.; Pack, J. D. Special points for brillouinzone integrations. Phys. Rev. B 1976, 13, 5188-5192.

[33] Heyed, J.; Scuseria, G. E. Efficient hybrid density functional calculations in solids: Assessment of the Heyd-ScuseriaErnzerhof screened Coulomb hybrid functional. J. Chem. Phys. 2004, 121, 1187-1193.

[34] Heyed, J.; Peralta, J. E.; Scuseria, G. E.; Martin, R. L. Energy band gaps and lattice parameters evaluated with the Heyd-Scuseria-Ernzerhof screened hybrid functional. $J$. Chem. Phys. 2005, 123, 174101-174108.

[35] Gaussian 09, Revision A.1, Frisch, M. J.; Trucks, G. W.; Schlegel, H. B.; Scuseria, G. E.; Robb, M. A.; Cheeseman, J. R.; Scalmani, G.; Barone, V.; Mennucci, B.; Petersson, G. A., et al. Gaussian, Inc., Wallingford CT, 2009.

[36] Huang, S. P.; Xu, H.; Bello, I.; Zhang, R. Q. Surface passivation-induced strong ferromagnetism of zinc oxide nanowires. Chem. Eur. J., in press, 2010, DOI: 10.1002/chem.201001167.

[37] Henkelman, G.; Uberuaga, B. P.; Jonsson, H. A climbing image nudged elastic band method for finding saddle points and minimum energy paths. J. Chem. Phys. 2000. 113, 9901-9904.

[38] Henkelman, G.; Jonsson, H. Improved tangent estimate in the nudged elastic band method for finding minimum energy paths and saddle points. J. Chem. Phys. 2000. 113, 9978-9985. 